\title{
ADONIS observations of hard X-ray emitting late B-type stars in Lindroos systems ${ }^{\star}$
}

\author{
N. Huélamo ${ }^{1}$, W. Brandner ${ }^{2,3}$, A. G. A. Brown ${ }^{3}$, R. Neuhäuser ${ }^{1,2}$, and H. Zinnecker ${ }^{4}$ \\ 1 Max-Planck-Institut für extraterrestrische Physik, Giessenbachstrasse 1, 85741 Garching, Germany \\ 2 University of Hawaii, Institut for Astronomy, 2680 Woodlawn Dr., Honolulu, HI 96822, USA \\ 3 European Southern Observatory, Karl Schwarzschildstrasse 2, 85748 Garching, Germany \\ 4 Astrophysikalisches Institut Potsdam, An der Sternwarte 16, 14482 Potsdam, Germany
}

Received 16 March 2001 / Accepted 3 May 2001

\begin{abstract}
We present adaptive optics $J H K_{\mathrm{S}}$ imaging observations of three main-sequence late B-type stars listed in the Lindroos Catalogue: HD 123445, HD 127971 and HD 129791. Given their spectral types, these stars should not be X-ray emitters. However, they have been detected by ROSAT and their X-ray emission has been attributed to possible unresolved late-type companions. We have carried out near-IR observations with ADONIS at the ESO $3.6 \mathrm{~m}$ but have not detected any late-type companions close to HD 127971 and HD 129791. This result leads us to conclude that either (i) they are spectroscopic binaries with unresolved low-mass companions, or (ii) they are intrinsic X-ray emitters. While the former case would be consistent with the reported high multiplicity of early-type (A and B) stars, the latter would yield a revision of stellar activity theories which do not predict X-ray emission from these stars. On the other hand, HD 123445 does indeed show visual companions, namely an apparent subarcsecond faint $\left(K_{\mathrm{s}} \sim 10\right)$ binary system at a projected separation of $5^{\prime \prime}$ from the late-B type star. The $J H K_{\mathrm{S}}$ magnitudes and colors of the components are consistent with (i) a pair of Pre Main Sequence (PMS) K-type stars at 140 pc (i.e. possible members of the Upper Centaurus Lupus association), (ii) a pair of Main Sequence M-type stars at $60 \mathrm{pc}$ and (iii) a pair of K-type giants at $2.6 \mathrm{kpc}$. While in the first case the reported X-ray emission can be ascribed to the new objects, in the second and third case it cannot, and we have to assume the late B-type star to be either a spectroscopic binary itself or a single star with intrinsic X-ray emission. Spectroscopy is required to confirm the possible PMS nature of the new binary and Chandra X-ray high spatial resolution (astrometric) imaging observations are required to definitely determine the source of the X-ray emission. If the B9 star results to be the X-ray emitter, near-IR spectroscopy can be used to investigate the presence of a T Tauri like spectroscopic companions.
\end{abstract}

Key words. stars: early-type - stars: binaries - infrared: stars - X-rays: stars

\section{Introduction}

Lindroos systems are defined as visual binaries mainly comprised of Main Sequence (MS) early-type primaries and later-type secondaries (Lindroos 1985). The ages of the primaries have been derived trough photometric and spectroscopic observations, showing values of the order of $\sim 10^{7} \mathrm{yr}$. Because this age is comparable to the contraction time scale of late-type stars to the MS, if the systems are bound the secondaries can be Pre-Main Sequence (PMS) stars still contracting to the Zero Age Main Sequence

Send offprint requests to: N. Huélamo,

e-mail: huelamo@mpe.mpg.de

* Based on observations collected at the European Southern Observatory, La Silla, under project 65.H-0568(A).
(ZAMS), that is, T Tauri (TTS) or Post-T Tauri stars (PTTS).

Pallavicini et al. (1992) and Martín et al. (1992) have reported the presence of youth (Li I absorption line) and activity ( $\mathrm{H} \alpha$ emission line) indicators among the late-type secondaries. Because the X-ray emission is another indicator of youth/activity among late-type stars (Neuhäuser et al. 1995), a study of the X-ray properties of the Lindroos secondaries has been undertaken to confirm their suspected PMS nature. The X-ray emission from Lindroos systems has been studied by Huélamo et al. (2000), as an extension of the work by Schmitt et al. (1993). The study was based on ROSAT High Resolution Imager (HRI) data, which was the only instrument on board the ROSAT satellite capable of resolving most of the systems. The analysis of the HRI X-ray data from 22 binary systems shows that 
16 of the resolved late-type secondaries are X-ray emitters, with X-ray luminosities and X-ray spectral energy distributions comparable to those from PMS late-type stars (Neuhäuser et al. 1995).

Surprisingly, the X-ray detection of several late Btype stars in Lindroos systems has been also reported. Theoretically, late B-type and early A-type stars are not expected to be X-ray emitters. Unlike O and early B-type stars, they do not show strong winds, so the X-rays cannot be produced by energetic shocks due to instabilities in the radiatively driven winds (Lucy \& White 1980; Owocki 1988) and, in contrast to late-type stars, they do not have a convective zone able to power a corona. Nevertheless, the X-ray emission from late-B and early-A type stars as seen by Einstein and ROSAT satellites has been reported by several authors (Caillault \& Zoonematkermani 1989; Caillault et al. 1994; Berghöfer \& Schmitt 1994; Simon et al. 1995; Berghöfer et al. 1997; Panzera et al. 1999). The most widely accepted explanation for their X-ray emission is the presence of unresolved late-type companions responsible for the X-ray detections. However, the moderate spatial resolution of the Einstein and ROSAT X-ray detectors has not allowed one to probe this hypothesis, and the intrinsic X-ray emission of late B-type stars is still an open question.

In the case of early-type stars in Lindroos systems, Huélamo et al. (2000) have found that 3 (out of 5) late B-type primaries show X-ray properties very different in comparison with similar MS late B-type stars. Firstly, they show large X-ray luminosities comparable to those from PMS late-type stars. Secondly, the spectral distribution of their X-ray emission is similar to that from TTS in star forming regions, that is, they are hard X-ray emitters. The fact that these X-ray properties are also similar to those from the Lindroos PMS late-type secondaries, has strengthened the hypothesis of unresolved PMS late-type companions to these 3 B-type primaries.

In order to check this hypothesis we have carried out diffraction limited IR observations of the 3 late B-type stars in Lindroos systems with a higher probability of showing close companions. Our main aim is to check if their X-ray emission can be ascribed to unknown latetype stars, previously unresolved. If so, this result would strengthen the idea that late B-type stars are not intrinsic $\mathrm{X}$-ray emitters. If not, it would suggest these late B-type stars are (i) spectroscopic binaries with even closer latetype companions or (ii) intrinsic X-ray sources. Note that an extended work on adaptive optics (AO) observations of $\mathrm{X}$-ray emitting late B-type stars has been recently carried out by Hubrig et al. (2001) on different targets.

The main properties of the three late B-type stars are described in Sect. 2, while the details of the data reduction are provided in Sect. 3. The results for each source are discussed in Sect. 4. The main conclusions are summarized in Sect. 5.

\section{X-ray properties of the late B-type stars under study}

The three stars under study are included in the so-called Lindroos catalogue (Lindroos 1985), and have late-type visual companions. All of them have been detected and resolved by the ROSAT High Resolution Imager (HRI), showing unusual X-ray properties in comparison with similar MS early-type stars.

As shown in Huélamo et al. (2000), MS early-type stars in Lindroos systems show X-ray luminosities which decrease with later spectral types. While early B-type stars show X-ray emissions comparable to that from PMS objects, late B-type and A-type stars show X-ray luminosities of $\log L_{\mathrm{x}}(\mathrm{erg} / \mathrm{s}) \sim 28$. The three late B-type stars under study in the present paper show X-ray luminosities larger than $\log L_{\mathrm{x}}(\mathrm{erg} / \mathrm{s})>29.5$, that is, clearly comparable to that from TTS.

Spectral information of the X-ray emission can be also derived through the so-called hardness ratio (HR). The HRI pulse height distribution can be used to compute a two-band (soft and hard) hardness ratio (see Huélamo et al. 2000 for details). The derived $H R$ for the sample of Lindroos primaries shows that they are generally soft X-ray emitters with $H R<0$, while the PMS late-type secondaries are hard X-ray sources with $H R>0$. In the case of the three late B-type stars with X-ray emission, their $H R$ is similar to that from young late-type stars (see Table 1).

Also note that a mean ratio of $\log L_{\mathrm{x}} / L_{\mathrm{bol}} \sim-6$ has been derived for late B-type stars observed in the $R A S S$ (Berghöfer et al. 1997). This value is two orders of magnitude larger than the $\log L_{\mathrm{x}} / L_{\mathrm{bol}} \sim-8$ value derived by Cassinelli et al. (1994) for stars with spectral types later than $\sim$ B2. Berghöfer et al. (1997) have attributed such a large ratio to possible late-type companions not resolvable in the RASS and responsible of the X-ray detection. The three Lindroos late-B type stars under study show $\log L_{\mathrm{x}} / L_{\mathrm{bol}}$ ratios between -4.5 and -6 , so they show large values that could certainly be related with the presence of X-ray emitting unresolved companions.

Therefore, our AO analysis is focused on the three late B-type Lindroos primaries stars resolved by the ROSAT HRI showing high X-ray luminosities and $H R$ typical of late-type stars, that is, we will study those late B-type stars with a higher probability of having an unresolved PMS late-type companion. Table 1 shows the stellar data for the three selected sources. Columns 1 and 2 provide the HD number and the spectral type of the stars. Columns 35 show the visual magnitude, the visual extinction to the source and the stellar mass (according to Lindroos 1985), respectively. The distance to the source and its proper motion, both derived from Hipparcos data, are provided in Cols. 6 and 7. The OB-association to which the stars probably belong (de Zeeuw et al. 1999) is given in Col. 8. Note that in the case of HD 127971, we have adopted the result by de Zeeuw et al. (1999) who do not find clear evidence of membership (to the Upper Centaurus Lupus 
Table 1. Main properties of the three Lindroos late B-type stars observed with ADONIS.

\begin{tabular}{llcccccccc}
\hline Star & Sp. T. & $\begin{array}{c}V \\
(\mathrm{mag})\end{array}$ & $\begin{array}{c}A_{v}^{1} \\
(\mathrm{mag})\end{array}$ & $\begin{array}{c}\text { Mass }^{1} \\
\left(M_{\odot}\right)\end{array}$ & $\begin{array}{l}\text { Distance }^{2} \\
(\mathrm{pc})\end{array}$ & Membership $^{3}$ & $\begin{array}{c}\mu_{\alpha}^{*}, \mu_{\delta}{ }^{4} \\
(\mathrm{mas})\end{array}$ & $\begin{array}{c}L_{\mathrm{x}}{ }^{5} \\
\left(10^{30}\right. \\
\mathrm{erg} / \mathrm{s})\end{array}$ & $\begin{array}{c}\mathrm{HR}^{5} \\
\text { HD 123445 }\end{array}$ \\
\hline B9 & 6.19 & 0.12 & 3.0 & $218 \pm 37$ & Upper Cen Lup & $-19.3 \pm 0.8,-17.4 \pm 0.6$ & $3.47 \pm 0.51$ & $0.19 \pm 0.13$ \\
HD 127971 & B7 & 5.89 & 0.08 & 3.5 & $109 \pm 9$ & & $-21.6 \pm 0.8,-23.2 \pm 0.7$ & $0.55 \pm 0.08$ & $0.49 \pm 0.13$ \\
HD 129791 & B9.5 & 6.94 & 0.26 & 2.5 & $129 \pm 16$ & Upper Cen Lup $-26.1 \pm 1.1,-20.8 \pm 0.8$ & $3.80 \pm 0.29$ & $0.56 \pm 0.07$ \\
\hline
\end{tabular}

Notes: ${ }^{1}$ Extracted from Lindroos (1985); ${ }^{2}$ Derived from Hipparcos parallaxes; ${ }^{3}$ de Zeeuw et al. $(1999) ;{ }^{4}$ Hipparcos proper motions; ${ }^{5}$ Data from Huélamo et al. (2000); the X-ray luminosities are derived using Hipparcos distances.

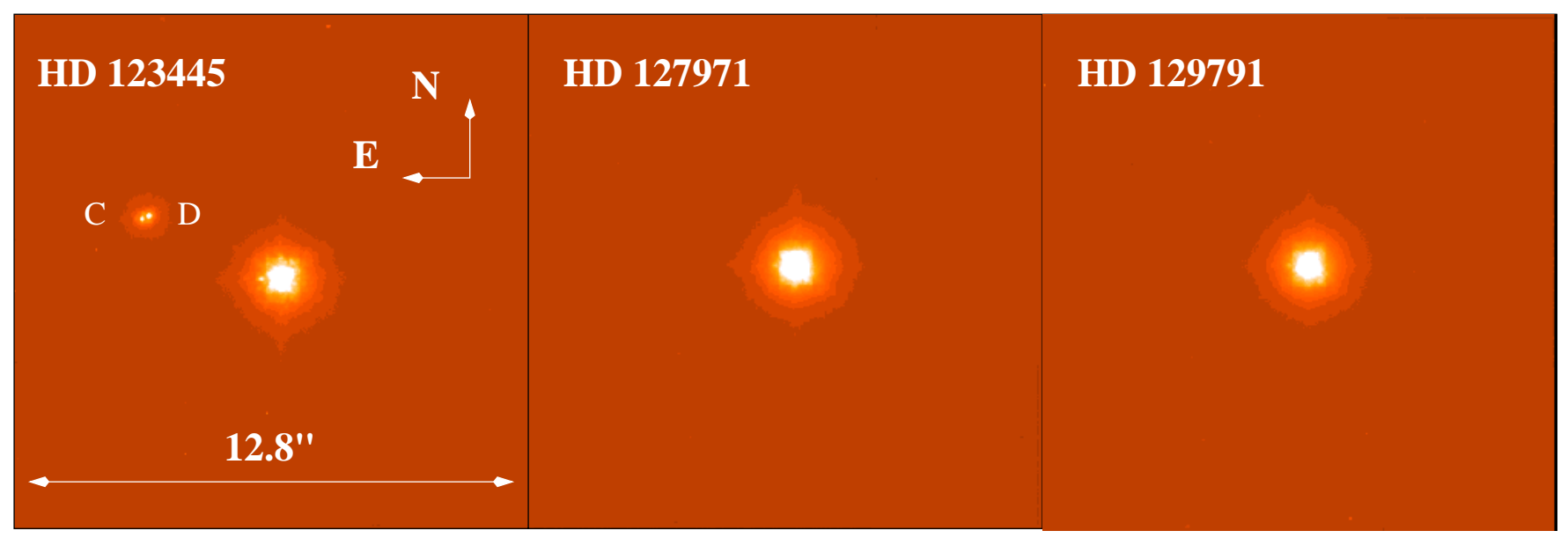

Fig. 1. $K_{\mathrm{S}}$-band images of the three stars observed with ADONIS. The whole field of the SHARP II camera $\left(12.8^{\prime \prime} \times 12.8^{\prime \prime}\right)$ is shown. As can be seen, only in the case of HD 123445 two new objects are detected at $\sim 5^{\prime \prime}$ NE from the central star (perhaps a true $0.25^{\prime \prime}$ binary system in the UCL association). In the case of HD 129791 and HD 127971 no extra sources are detected.

association) of this star based on Hipparcos data. Finally, the derived X-ray luminosity of the sources together with their $H R$ (Huélamo et al. 2000) are provided in Cols. 9 and 10 .

\section{The ADONIS data}

The IR data of the three late B-type stars were obtained with the Adaptive Optics Near Infrared System (ADONIS) plus the System for High Angular Resolution Pictures (SHARP) II camera at the $3.6 \mathrm{~m}$ telescope at La Silla Observatory. The main advantage of the AO system is that provides diffraction limited resolution in the core of the Point Spread Function (PSF) (i.e. 0.15" in the $K$-band). The observations were carried out on the 2 nd June 2000, in the course of the programme 65.H-0658(A).

The three targets are brighter than 8 mags in the optical (see Table 1) so the Reticon wave front sensor was used to perform the atmospheric correction. The observations were carried out in the $J H K_{\mathrm{S}}$ filters. For each source, 120 individual frames (30 frames each at 4 different positions of the detector) were taken in the three filters. The individual exposure times range between 0.3 and $0.8 \mathrm{~s}$, and were chosen so as to avoid saturating the detector. Several PSF-calibrator and standard stars were also observed during the night.
Note that the three late B-type stars under study have known visual companions located at more than $15^{\prime \prime}$ (see Lindroos 1986). Because the field of view (FOV) of the SHARP II camera is $12.8^{\prime \prime} \times 12.8^{\prime \prime}$ (with a plate scale of $0.05^{\prime \prime} /$ pixel), these companions are located outside the frames.

The data reduction was performed using the Eclipse (Devillard 1997) and the IRAF packages. All the images are sky-subtracted, dark and flat-field corrected. A badpixel map correction was also applied. The final images are the result of averaging the individual frames with higher quality, i.e. with larger Strehl ratio (SR) and smaller $F W H M$. This was necessary due to the rapid seeing variations during the night (from $0.7^{\prime \prime}$ to $1.6^{\prime \prime}$ ).

We have computed the limiting magnitudes of the final frames in each filter, assuming a $3 \sigma$ level over the background as a detection. We have derived these magnitudes at three different positions from the central star, namely $0.5^{\prime \prime}, 1^{\prime \prime}$ and $2^{\prime \prime}$, in order to gain a better understanding of the detection limits. Note that the resulting magnitudes are dependent of several factors: the brightness of the central object, the Strehl ratio, the number of averaged individual images and the individual exposure time which makes the readout noise (RON) an important limitation.

Kirkpatrick \& McCarthy (1994) provide absolute near-IR magnitudes of M-dwarfs down to the substellar limit (M7 to M9 dwarfs). Using these values, we have 
Table 2. Observational data of the 3 stars under study.

\begin{tabular}{llllllllll}
\hline HD & Band & \multicolumn{2}{c}{$\begin{array}{c}\text { Strehl ratio } \\
\text { Mean }\end{array}$} & Min. ${ }^{1}$ & Max. & No. frames & Exp. time & \multicolumn{3}{c}{ Limiting magnitudes } \\
& & & & & & & $0.5^{\prime \prime}$ & $1^{\prime \prime}$ & $2^{\prime \prime}$ \\
& & & & & & $(\mathrm{sec})$ & $(\mathrm{mag})$ & $(\mathrm{mag})$ & $(\mathrm{mag})$ \\
\hline \multirow{2}{*}{123445} & $J$ & 6 & 5 & 7.2 & 38 & 0.5 & 14.2 & 16.2 & 19.5 \\
& $H$ & 14 & 10 & 16 & 73 & 0.3 & 13.1 & 16.5 & 19.4 \\
& $K_{\mathrm{S}}$ & 28 & 25 & 32 & 61 & 0.5 & 15.1 & 16.3 & 18.9 \\
& $J$ & 1.4 & 1 & 2.2 & 64 & 0.6 & 14.5 & 16.2 & 18.6 \\
& $H$ & 5 & 3 & 8 & 34 & 0.6 & 14.3 & 15.8 & 18.6 \\
& $K_{\mathrm{S}}$ & 45 & 30 & 52 & 100 & 0.4 & 13.8 & 16.6 & 19.1 \\
& $J$ & 3 & 2.5 & 4 & 21 & 0.8 & 14.7 & 16.9 & 19.5 \\
& $H$ & 7 & 5 & 12 & 51 & 0.8 & 15.0 & 16.9 & 19.8 \\
& $K_{\mathrm{S}}$ & 20 & 15 & 24 & 56 & 0.6 & 15.1 & 17.4 & 19.8 \\
\hline
\end{tabular}

Notes: ${ }^{1}$ Minimum Strehl ratio selected to improve the quality of the final images.

derived the expected near-IR magnitudes of late M-dwarfs at the distances of our three targets (that is, 218, 109 and $129 \mathrm{pc}$ ). These data, together with the limiting magnitudes provided in Table 2 show that we could detect companions around the three sources down to a spectral type of M9 at projected separations larger than $1^{\prime \prime}$.

The observational data are summarized in Table 2 . The name of the star and the filter are provided in Cols. 1 and 2. The minimum SR selected for each of the final frames, together with the maximum and mean values, are given in Cols. 3-5. The total number of frames (out of a total of 120) used for the final averaging and their individual exposure times are given in Cols. 6 and 7. The limiting magnitudes for each of the final frames at $0.5^{\prime \prime}$, $1^{\prime \prime}$ and $2^{\prime \prime}$ from the late B-type star are provided in the last columns of Table 2 .

\section{Analysis of the data}

We have derived the near-IR magnitudes of all the detected objects after carrying out aperture photometry of the sources. The selected aperture radius for all the targets is 50 pixels $\left(2.5^{\prime \prime}\right)$. The zero-points in the three filters were derived using standard stars observed during the night. We obtained the following values: $21.5,21.9$ and 22.2 mags, in the $K_{\mathrm{S}}, H$ and $J$-band, respectively. The mean extinction coefficients at La Silla for the three filters were used. The main uncertainties of the IR magnitudes come from the errors associated to the zero points and the extinction coefficients in each band, resulting in a typical error of $\pm 0.1 \mathrm{mag}$ in the three filters.

The analysis of the final images shows that in the case of HD 123445 two new sources have been detected at $5^{\prime \prime}$ NE from the B9 star. As it is seen in Fig. 1, the two objects are very close to each other $\left(0.26^{\prime \prime} \pm 0.01\right)$, so their IR magnitudes were determined through multi-aperture photometry. In the case of HD 127971 and HD 129791 no extra objects were detected in the field.

The derived near-IR magnitudes for the 5 detected objects are listed in Table 3. In the case of the late B-type stars, they are in good agreement with those reported by Lindroos (1983). Also the derived IR photometric distances are in good agreement with the Hipparcos distances, allowing for an error of one subtype in the spectral types.

The $J-H$ and $H-K_{\mathrm{S}}$ colors have been computed for the 5 objects. Those are represented in the color-color diagram shown in Fig. 2. We will discuss the results for each star in the following subsections.

\section{1. $H D 123445$}

HD 123445 is a B9 type star with a known visual companion, $B$, at a separation of $28.6^{\prime \prime}$. In our observations, we have detected two new sources in the field of the B9 star. The two objects ( $C$ and $D$ in Fig. 1) are located at $4.58 \pm 0.01^{\prime \prime} \mathrm{NE}$ and $4.46 \pm 0.01^{\prime \prime} \mathrm{NE}$ from HD 123445, respectively. Their position angles (PA), measured from the north to the east, are $\operatorname{PA}(C)=65 \pm 1^{\circ}$ and $\operatorname{PA}(D)=64 \pm 1^{\circ}$. From the Hipparcos parallax, the distance to HD 123445 is 218 pc. Hence, the projected separation of these stars with respect to the B9 primary is $\sim 1000$ AU. Note, however, that the uncertainty in the Hipparcos distance is large, so the star could be located much closer $(181 \mathrm{pc})$ or much further $(255 \mathrm{pc})$ in the association. Given that the mean distance to the UCL association is $140 \mathrm{pc}$ with a depth of $\pm 50 \mathrm{pc}$ (de Bruijne et al. 1999), it is most probable that HD 123445 is at the back end of the association with a distance between 180 and 190 pc.

As a first step, we have studied the ROSAT HRI image of HD 123445 to check if the position of the single X-ray detection is consistent with the optical position of the new binary. Figure 3 shows the ROSAT HRI X-ray image of HD 123445. The nominal positional error of the HRI is $5^{\prime \prime}$, although a bore-sight correction as much as $10^{\prime \prime}$ can be applied. Therefore, we have plotted the position of the X-ray detection enclosed by a circle of $10^{\prime \prime}$ radius. The Hipparcos optical position of HD 123445 (open triangle) together 
Table 3. Near-IR photometric data of the 5 objects detected

\begin{tabular}{lrrrcc}
\hline Star & $\begin{array}{r}J \\
(\mathrm{mag})\end{array}$ & $\begin{array}{r}H \\
(\mathrm{mag})\end{array}$ & $\begin{array}{r}K_{\mathrm{S}} \\
(\mathrm{mag})\end{array}$ & $\begin{array}{c}J-H \\
(\mathrm{mag})\end{array}$ & $\begin{array}{c}H-K_{\mathrm{S}} \\
(\mathrm{mag})\end{array}$ \\
\hline HD 123445 & 6.2 & 6.2 & 6.2 & 0.0 & 0.0 \\
Comp. $C$ & 10.8 & 10.1 & 9.9 & 0.7 & 0.2 \\
Comp. $D$ & 10.7 & 10.1 & 9.9 & 0.6 & 0.2 \\
HD 127971 & 6.0 & 5.9 & 5.9 & 0.1 & 0.0 \\
HD 129791 & 6.7 & 6.6 & 6.6 & 0.1 & 0.0 \\
\hline
\end{tabular}

Note: The errors in the magnitudes are \pm 0.1 in the three bands.

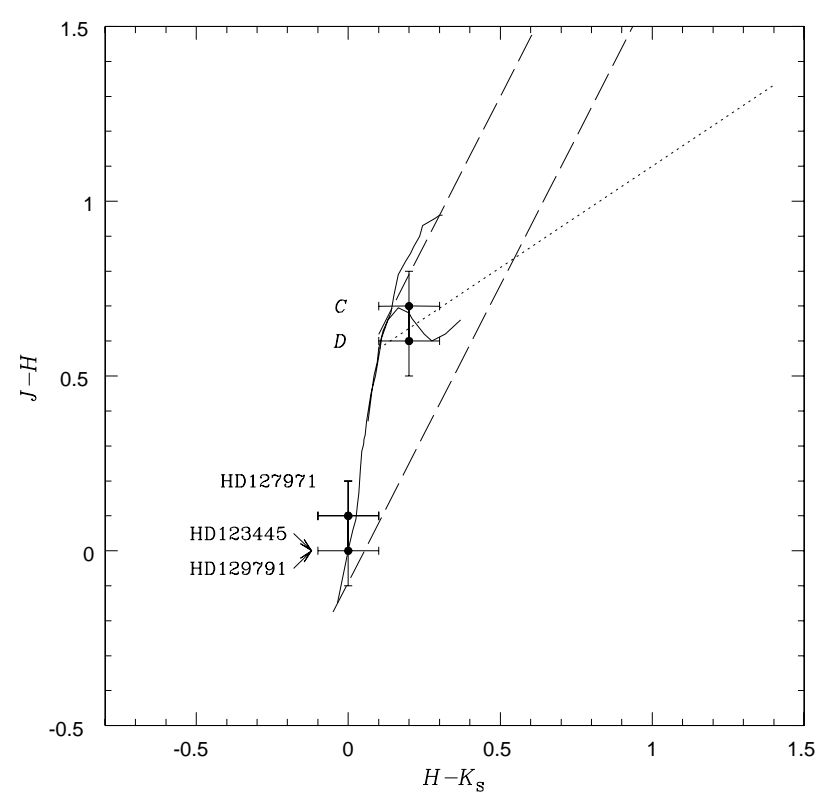

Fig. 2. Color-color diagram for the 3 stars under study. We have represented by solid lines the unreddened main sequence and giant sequence from Bessell \& Brett (1988). The dashed lines represent the reddening vectors (Rieke \& Lebofsky 1985) while the dotted line represents the color-color relation followed by WTTS and CTTS in Taurus (Meyer et al. 1997). The three late B-type stars lie at the left bottom of the diagram. The two sources located at $5^{\prime \prime} \mathrm{SE}$ from HD 123445 lie in the upper region of the diagram close to the K-giants and the MS M-type stars. These sources also followed the color-color relation found for PMS late-type stars, CTTS and WTTS, in Taurus (Meyer et al. 1997) within the errors.

with the optical position of the two new sources (open hexagon) are over-plotted. As noted in Huélamo et al. (2000), the single X-ray detection is displaced 7.7" from the optical position of HD 123445 . However, the new binary is certainly closer to the X-ray detection than the late B-type star, being located at $4.2^{\prime \prime} \mathrm{SW}$ from the X-ray source.

Apart from HD 123445, there are no other bright Xray sources in the FOV of the HRI, so it is not possible to measure relative positions through X-ray field objects, i.e. we could not do a proper bore-sight correction. Hence, we can firstly conclude that although the X-ray detection is closer and therefore possibly related to the new objects $(C$ and $D)$, it could also be ascribed to HD 123445 itself.

As a second step, we have analyzed the near-IR data from the detected binary. The IR magnitudes of HD 123445 together with those from the two new objects are listed in Table 3. It can be seen that the two members of the new detected binary show very similar IR magnitudes and colors. When we study the position of the two faint objects in a color-color diagram (Fig. 2), we can see that within the errors both lie close to the region of the MS and PMS M and K-type stars. Their colors are also consistent with those from giant K-type stars. We will analyze each of these possibilities to study if any of them is in good agreement with the derived X-ray data:

a) Both objects show IR colors consistent with M0V to M5V stars. Therefore, we will assume an intermediate spectral type of M2 for both sources.

According to Kirkpatrick \& McCarthy (1994), the absolute $H$-magnitude of a M2V star is $M_{H}=6.21$. Using the derived $H$-magnitudes of our objects and the distance modulus relation, we can derive a photometric distance of $60 \mathrm{pc}$. In this case, the objects would not be bound to HD 123445. If they were so close, they would not belong to any known star forming region, so we will assume them to be MS M-type stars but no PMS objects. However, this assumption will be discussed later.

Gliese \& Jahreiß (1991) and Henry et al. (1994) have identified a large sample of MS low-mass stars in the solar neighborhood $(d<25 \mathrm{pc})$. The X-ray emission of these nearby MS late-type stars has been studied by Schmitt et al. (1995), Schmitt (1997) and Hünsch et al. (1999) based in ROSAT data. The mean/median X-ray luminosity reported for MV type stars is $L_{\mathrm{x}}(\mathrm{erg} / \mathrm{s}) \sim$ $\left.5.5 \times 10^{28} / 8.6 \times 10^{27}\right)$, with a large dispersion of $\sigma \sim$ $1.3 \times 10^{29} / 2.2 \times 10^{28}$, respectively.

Making use of the mean X-ray luminosity (in order to be conservative and to include the strongest X-ray emitters), we can derive the expected ROSAT HRI count rate from a M-type star at $60 \mathrm{pc}$. The X-ray luminosity of a source is given by the expression:

$L_{\mathrm{x}}=\frac{4 \pi d^{2}}{E C F} \times($ rate $)$

where $d$ is the distance to the source, $E C F$ the energyconversion-factor and rate the X-ray counts per second coming from the source. The ECF depends on the detector response and the underlying model for the X-ray spectrum. In our case, we have assumed a RaymondSmith thermal spectrum with temperature $k T=1 \mathrm{keV}$ (typical for late-type stars), and a negligible extinction to the source $\left(\log N_{\mathrm{H}}\left(\mathrm{cm}^{-2}\right)=19.0\right)$. In accordance with the HRI response curve, the derived $E C F$ is $0.20 \times$ $10^{11} \mathrm{cts} \mathrm{cm}^{-2} \mathrm{erg}^{-1}$. Following Eq. (1), the expected HRI count rate for a M-type star at $60 \mathrm{pc}$ is rate $(\mathrm{cts} / \mathrm{s})=$ 0.002 . We have detected two objects in the near-IR images. If we assume they emit the same amount of X-rays, the total expected count rate would be $0.004 \mathrm{cts} / \mathrm{s}$. However, when studying the HRI X-ray detection of HD 123445, the 


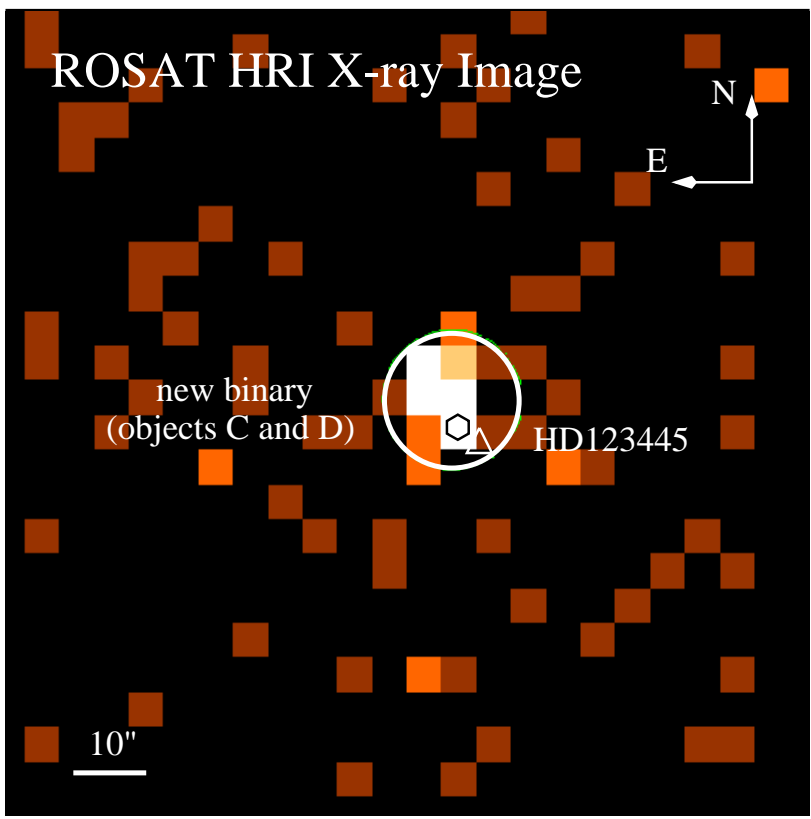

Fig. 3. ROSAT X-ray image of HD 123445 . The X-ray source is located inside the white circle, whose radius is $10^{\prime \prime}$, that is, it represents the maximum positional error of the ROSAT HRI. The optical positions of HD 123445 and the new binary ( $C$ and $D$ objects) are represented by an open triangle and a hexagon, respectively. As seen, the new subarcsecond binary lie closer to the X-ray detection than the late B-type star. However, both positions are within the HRI positional error, so the X-ray emission could be ascribed to any of them.

derived count rate is rate $(\mathrm{cts} / \mathrm{s})=0.012 \pm 0.002$, which is larger than that expected from two MS M-type stars. Note that the X-ray emission from HD 123445 was shown to be from a quiescent, non-flaring source (Huélamo et al. 2000).

The large scatter in the X-ray luminosities of MS Mtype stars suggests very different properties among the sample, and does not allow us to clearly conclude that the new objects are inconsistent with this spectral type. As a further test, we have made use of the empirical saturation limit of $\log \left(L_{\mathrm{x}} / L_{\mathrm{bol}}\right)=-3$ for late-type stars to compare it with that derived for our stars. The bolometric luminosity for a MS M2-type star at $60 \mathrm{pc}$ (using the bolometric correction from Schmidt-Kaler 1982), results in $L_{\mathrm{bol}}(\mathrm{erg} / \mathrm{s})=1.42 \times 10^{32}$. If we suppose that all the Xrays $(0.012 \mathrm{cts} / \mathrm{s})$ are emitted only by one of the objects, and assuming an ECF as for late-type coronal sources $\left(0.20 \times 10^{11} \mathrm{cts} \mathrm{cm}^{-2} \mathrm{erg}^{-1}\right)$ the derived $\log \left(L_{\mathrm{x}} / L_{\mathrm{bol}}\right)$ ratio is larger than the derived saturation limit of -3 for late-type stars. On the other hand, if we assume that each of the two objects emits half of the X-rays, the derived X-ray luminosity assuming the same ECF would be $L_{\mathrm{x}}(\mathrm{erg} / \mathrm{s})=1.29 \times 10^{29}$. Then, the derived $\log \left(L_{\mathrm{x}} / L_{\mathrm{bol}}\right)$ ratio for both objects is -3.04 , that is, similar to the saturation limit of -3 . This implies that both objects must be very active M-type stars. However, according to Schmitt et al. (1996) the X-ray emission from MS late-type stars in the solar neighborhood seems to be generally moder- ate. In fact, most of the MS M-type nearby stars studied by Hünsch et al. (1999) show negative $H R$, that is, they are soft X-ray sources mainly due to their lower levels of activity and because of negligible interstellar absorption at small distances. Therefore, the probability of having two very active MS M-type stars at $60 \mathrm{pc}$ seems to be low according to these $\mathrm{X}$-ray studies.

Since young late-type objects in the solar neighborhood also seem to be abundant (Sterzik \& Schmitt 1997), we could also have assumed both objects to be PMS Mtype stars. Although there are only two M-type stars among the sample of most active nearby young stars, it seems that their X-ray emission is two orders of magnitude higher than in MS M-type stars. However, their $H R$ is generally negative in part because of the negligible interstellar absorption. This absorption is not significant at $60 \mathrm{pc}$. In fact, Stelzer \& Neuhäuser (2000) show that most of the young late-type stars which belong to the nearby Tucanae and TW Hya associations $(d \sim 50 \mathrm{pc}$, age $\sim 10 \mathrm{Myr})$ show negative $H R$. Therefore, even if the new objects were young M-type stars, this would not be consistent with our derived $H R$, which is more common from young objects in star forming regions (Neuhäuser et al. 1995).

b) We will now assume both objects to be K2-type stars. If we follow the same line of reasoning as before, and use the absolute visual magnitudes from Schmidt-Kaler (1982), we can derive a photometric distance of $137 \mathrm{pc}$. This value is very close to the derived distance to the UCL association $(140 \mathrm{pc})$, so we will assume the new binary to be a member of this group. Note that, as in the previous case, both objects would not be bound to the late B-type star.

According to de Geus et al. (1989) the estimated age of the UCL association is 13 Myr. That means that our two K-type stars could be young objects still contracting to the MS, that is, they might be T Tauri stars. As can be seen from their IR colors, they do not show significant IR excesses. Hence, they could be considered as Weakline T Tauri stars (WTTS), that is, young stars without optically thick accretion disks. In fact, we show in Fig. 2 that both objects follow the color-color relation

$J-H=(0.58 \pm 0.11) \times(H-K)+0.52 \pm 0.06$

found by Meyer et al. (1998) for WTTS and CTTS in Taurus, within the uncertainties.

The typical X-ray emission from K-type WTTS in Taurus is $\log L_{\mathrm{x}}(\mathrm{erg} / \mathrm{s})=29.80 \pm 0.12$ (Stelzer \& Neuhäuser 2001). If we consider this luminosity, we can repeat the procedure described above assuming the new binary to be comprised of two K-type stars at $140 \mathrm{pc}$ emitting the same amount of X-rays. Hence, the derived count rate for the ROSAT HRI is rate $(\mathrm{cts} / \mathrm{s})=0.007$ for one object, and rate(cts/s) $=0.014$ for two similar ones. This value is certainly in very good agreement with the computed count rate for HD 123445 (rate $(\mathrm{cts} / \mathrm{s})=$ $0.012 \pm 0.002)$. Note that this result would imply the need of two objects at the same distance to produce the observed X-ray luminosity. 
Repeating the same procedure as in case (a) we have derived the bolometric luminosity of a K2-type star at $137 \mathrm{pc}$, obtaining a value of $L_{\mathrm{bol}}(\mathrm{erg} / \mathrm{s}) \sim 9.8 \times 10^{32}$. If we assume that just one of the sources emits all the X-rays, the derived X-ray luminosity at $137 \mathrm{pc}$ is $\log L_{\mathrm{x}}(\mathrm{erg} / \mathrm{s})=1.34 \times 10^{30}$. In this case the $\log \left(L_{\mathrm{x}} / L_{\mathrm{bol}}\right)$ ratio is larger than the saturation limit of -3 for late-type stars. However, if we assume that each of the sources emits half of the X-rays, the derived $\log \left(L_{\mathrm{x}} / L_{\mathrm{bol}}\right)$ ratio is equal to $7 \times 10^{-4}$, that is, close to the saturation limit. If we take into account that the $\log \left(L_{\mathrm{x}} / L_{\mathrm{bol}}\right)$ ratio of TTS in Taurus ranges between -3 and -5 (Stelzer \& Neuhäuser 2001), the result is consistent with both stars being very active PMS objects.

Concerning the $H R$, most of the WTTS listed in Stelzer \& Neuhäuser show positives values, meaning that they are mainly hard X-ray sources. Therefore, both the X-ray luminosity and the $H R$ can be reproduced assuming that the X-ray emission from HD 123445 originates by two PMS K-type stars at $140 \mathrm{pc}$.

c) The final possibility under study is that both objects are background evolved stars, given that their color indexes are also compatible with $\mathrm{K}$-type giants (from K0 to K4). A K2 giant has typical colors of $V-K=2.70$, $J-H=0.63$ and $H-K=0.12$ and a visual absolute magnitude of $M_{v}=+0.5$ (Schmidt-Kaler 1982). Using these data and the distance modulus relation we can derive a photometric distance of $2.6 \mathrm{kpc}$ to both sources.

Late-type giants are easily detected at IR wavelengths given that their effective temperature $(4000 \mathrm{~K})$ and their extended atmospheres makes them strong emitters in this spectral range (see Alves et al. 1998 for further discussion). The Galactic coordinates of HD 123445 are $\ell\left(^{\circ}\right)=317.52$ and $b\left(^{\circ}\right)=17.20$, so we are studying a region relatively close to the galactic bulge, increasing the probability of detection of red giant stars.

On the other hand, late-type giant stars are known to be X-ray sources (Ayres et al. 1995; Hünsch et al. 1998). However, given their typical X-ray fluxes they could not have been detected at a distance of $2.6 \mathrm{kpc}$ by ROSAT. In this case, we would have to relate the reported X-ray emission to HD 123445 itself, implying that it would probably be a spectroscopic binary or an intrinsic X-ray emitter.

If we assume HD 123445 to be a spectroscopic binary, we can roughly estimate the spectral type of the supposed companion making use of the derived X-ray luminosity. As in Sect. 4, we will assume a Raymond-Smith thermal spectrum with a temperature of $1 \mathrm{keV}$. The $E C F$ is derived considering the $A_{V}$ listed in Table 1 . The result is that a late-type star at the same distance as HD 123445 would show an X-ray luminosity of $\log L_{\mathrm{x}}(\mathrm{erg} / \mathrm{s})=30.60$. This value agrees with that reported for PMS G-type stars in Taurus (Stelzer \& Neuhäuser 2001). Note, however, that in the case of HD 123445 there are no indications of a close companion in accordance with the Hipparcos Catalogue.

After the analysis of the three possibilities, it seems that the case of two PMS K-type stars is in good agreement with the derived X-ray data. However, we note that spectroscopy is required before drawing final conclusions about the nature of these two sources. In particular, the detection of the Li I (6708 $\AA$ ) absorption line would be a good indication of their PMS nature. Another important clue about the true distance of the subarcsecond pair could be derived from its orbital motion. Note that if the two objects are a true UCL binary, its orbital period would be $\sim 70 \mathrm{yr}$ (and $\sim 22 \mathrm{yr}$ for a foreground binary with two M-type stars at $60 \mathrm{pc}$ ). Therefore, we could easily detect its orbital motion carrying out new IR observations in a few years.

\section{2. $H D 127971$ and HD 129791}

In the case of HD 127971 and HD 129791 no extra sources have been detected in their surroundings (neither latetype stars nor substellar companions). This result implies that either (i) they are spectroscopic binaries with unresolved late-type stars or (ii) binary systems with substellar companions at a distances smaller than $1^{\prime \prime}$ or (iii) they are single B-type stars with intrinsic X-ray emission.

Note that both stars are not resolved into multiple systems by Hipparcos. However, the Hipparcos Catalogue provides an acceleration solution for HD 129791 which suggests the presence of an unresolved lower-mass companion. In fact, the possibility of having two spectroscopic binaries is supported by several studies showing that the multiplicity of OB stars is rather high (i.e. Abt 1983; Abt et al. 1990; Morrell \& Levato 1991; Quist \& Lindegren 2000).

We have roughly estimated the spectral types of the possible unresolved companions to these B-type stars, following the same procedure described above. In the case of HD 127971, the derived X-ray luminosity for a late-type star at the same distance would be $\log L_{\mathrm{x}}(\mathrm{erg} / \mathrm{s})=29.80$, which is consistent with a young K-type star (Stelzer \& Neuhäuser 2001). For HD 129791, the derived X-ray luminosity is $\log L_{\mathrm{x}}(\mathrm{erg} / \mathrm{s})=30.60$, which in good agreement with a PMS G-type star (as in the case of HD 123445). This X-ray luminosity is larger than that reported for HD $129791 \mathrm{~B}^{1}$ (a PMS K5-type star probably bound to the B9 primary star), which would be consistent with their difference in spectral types.

Note that if the lack of intrinsic X-ray emission from late B-type and early A-type stars could be demonstrated, then X-ray observations will be a suitable method for deriving the frequency of spectroscopic late-type companions among these intermediate-mass stars.

\section{Results and conclusions}

The ADONIS IR $J H K_{\mathrm{S}}$ observations of 3 Lindroos late Btype stars have allowed us to study the presence/absence of close visual companions around them which could

\footnotetext{
1 Note that there is a typo in the X-ray luminosity of HD129791 B in Table 5 of Huélamo et al. (2000), being the correct value $L_{\mathrm{x}}=(6.64 \pm 1.33) \times 10^{29} \mathrm{erg} / \mathrm{s}$.
} 
possibly explain their X-ray emission. Our main conclusions can be summarized as follows:

1. Neither HD 127971 nor HD 129791 show objects around them in the AO images, implying that close visual late-type companions are not the explanation for their $\mathrm{X}$-ray emission. Further investigation (near-infrared spectroscopy) is required in order to find out if these two stars are spectroscopic binaries with unresolved late-type companions or if they are intrinsic X-ray sources.

However, in the case of HD 123445 two new objects are detected close to the late B-type star. Although the nature of both sources can only be determined through spectroscopic measurements, near IR magnitudes and colors show that they are most probably PMS K-type stars at a distance of $140 \mathrm{pc}$ (projected towards but not physically bound to HD 123445). In that case, the derived photometric distance is in good agreement with that from the UCL association to which HD 123445 also belongs. Moreover, the analysis of the X-ray data suggests that they could certainly be responsible of the hard X-ray emission associated with HD 123445.

2. The X-ray data from HD 127971 and HD 129791 have allowed us to roughly estimate the spectral types of possible unresolved companions. In both cases, a young late-type star (a G-type and a K-type respectively) could be responsible for the X-ray emission. With these latetype companions (masses equal to or less than $1 M_{\odot}$ ), the mass ratio of the spectroscopic binaries would be smaller than 1.

If it can be established that early $\mathrm{A}$ and late-type $\mathrm{B}$ stars lack intrinsic X-ray emission, then a fruitful means of systematically searching for lower-mass binary companions around this class of primary stars (of order 3-4 $M_{\odot}$ ) would be to carry out snapshot Chandra observations of a sample of early A and late-type B-stars. The main goal would be to determine their binary frequency and their mass ratio distribution, and to see if their binary properties differ from those of more massive or lower mass primaries, in particular solar-type primaries (Duquennoy \& Mayor 1991).

Acknowledgements. We are grateful to F. Eisenhauer, L. Close M. Petr and B. Brandl for their assistance during the data reduction. Special thanks to J. Alves, B. König and G. Wuchterl for their useful comments. The ROSAT project is supported by the Max-Planck-Society and the German Goverment (DRL/BMBF). WB acknowledges support by NSF and NASA. RN wishes to acknowledge financial support from the Bundenministerium für Bildung und Forschung through the Deutsche Zentrum für Luft- und Raumfahrt e.V. (DLR) under grant number 50 OR 0003.

\section{References}

Abt, H. A. 1983, ARA\&A, 21, 343

Abt, H. A., Gómez, A. E., \& Levy, S. G. 1990, ApJS, 74, 551 Alves, J., Lada, C. J., Lada, E., Kenyon, S. J., \& Phelps, R. 1998, ApJ, 506, 292

Ayres, T. R., Fleming, T. A., Simon, T., et al. 1995, ApJS, 96, 223
Berghöfer, T. W., \& Schmitt, J. H. M. M. 1994, A\&A, 292, L5

Berghöfer, T. W., Schmitt, J. H. M. M., Danner, R., \& Cassinelli, J. P. 1997, A\&A, 322, 167

Bessell, M., \& Brett, J. 1988, PASP, 100, 1134

Caillault, J. P., \& Zoonematkermani, S. 1989, ApJ, 338, L57

Caillault, J. P., Gagne, M., \& Stauffer, J. R. 1994, ApJ, 432, 386

Cassinelli, J. P., Cohen, D. H., MacFarlane, J. J., Sanders, W. T., \& Welsch, B. Y. 1994, ApJ, 421, 705

de Bruijne, J. H. J. 1999, MNRAS, 310, 585

de Geus, E. J., de Zeeuw, P. T., \& Lub, J. 1989, A\&A, 216, 44 de Zeeuw, P. T., Hoogerwerf, R., de Bruijne, J. H. J, Brown, A. G. A., \& Blaauw, A. 1999, AJ, 117, 354

Devillard, N. 1997, The eclipse software, The messenger, No. 87

Duquennoy, \& Mayor, M. 1991, A\&A, 248, 485

Gliese, W., \& Jahreiß, H. 1991, Preliminary version of the third Catalogue of Nearby Stars, ed. L. E. Brotzmann, \& S. E. Gesser, The Astronomical data Center CD-ROM: selected Astronomical Catalogues, vol. 1; NASA Astronomical Data Center, Goddard Space Flight Center, Greenbelt, MD

Henry, T. J., Kirkpatrick, J. D., \& Simons, D. A. 1994, AJ, 108,1437

Hubrig, S., Le Mignant, D., North, P., \& Krautter, J. 2001, $\mathrm{A} \& \mathrm{~A}$, in press

Huélamo, N., Neuhäuser, R., Stelzer, B., Supper, R., \& Zinnecker, H. 2000, A\&A, 359, 227

Hünsch, M., Schmitt, J. H. M. M., \& Voges, W. 1998, A\&AS, 127,251

Hünsch, M., Schmitt, J. H. M. M., Sterzik, M. F., \& Voges, W. 1999, A\&AS, 135, 319

Kirkpatrick, J. D., \& McCarthy, D. W. 1994, AJ, 107, 333

Lindroos, K. P. 1983, A\&AS, 51, 161

Lindroos, K. P. 1985, A\&AS, 60, 183

Lindroos, K. P. 1986, A\&A, 156, 223

Lucy, L. B., \& White, R. L. 1980, ApJ, 241, 300

Martín, E. L., Magazzù, A., \& Rebolo, R. 1992, A\&A, 257, 186

Meyer, M. R., Calvet, N., \& Hillenbrand, L. A. 1997, AJ, 114, 288

Morrell, N., \& Levato, H. 1991, ApJS, 75, 965

Neuhäuser, R., Sterzik, M. F., Schmitt, J. H. M. M., Wichmann, R., \& Krautter, J. 1995, A\&A, 297, 391

Owocki, S. P., Castor, J. I., \& Rybicki, G. B. 1988, ApJ, 335, 914

Panzera, M. R., Tagliaferri, G., Passinetti, L., \& Antonello, E. 1999, A\&A, 348, 161

Pallavicini, R., Pasquini, L., \& Randich, S. 1992, A\&A, 261, 245

Quist, C. F., \& Lindegren, L. 2000, A\&A, 361, 770

Rieke, G. H., \& Lebofsky, M. J. 1985, ApJ, 288, 618

Schmitt, J. H. M. M. 1997, A\&A, 318, 215

Schmitt, J. H. M. M., Zinnecker, H., Cruddace, R., \& Harnden, F. R. Jr 1993, ApJ, 402, L13

Schmitt, J. H. M. M., Fleming, T. A., \& Giampapa, M. S. 1995, ApJ, 450, 392

Schmidt-Kaler, Th. 1982, in Landolt-Börnstein New Series, Group VI, vol. 2b, 1, ed. K. Schaifers, \& H. H. Voigt (Springer-Verlag)

Simon, T. Drake, S. A., \& Kim, P. D. 1995, PASP, 107, 1034

Stelzer, B., \& Neuhäuser, R. 2000, A\&A, 361, 581

Stelzer, B., \& Neuhäuser, R. 2001, A\&A, submitted

Sterzik, M., \& Schmitt, J. H. M. M. 1997, AJ, 114, 1673 